\title{
87-W, 1018-nm Yb-fiber ultrafast seeding source for cryogenic Yb:YLF amplifier
}

\author{
Yi Hua, ${ }^{1}$ Wei Liu, ${ }^{1}$ Michael Hemmer, ${ }^{1}$ Luis E. Zapata, ${ }^{1}$ Gengji Zhou, ${ }^{1}$ Damian N. Schimpf, ${ }^{1,2}$ Tino Eidam, \\ ${ }^{3,4}$ Jens Limpert, ${ }^{3,4}$ Andreas Tünnermann, ${ }^{3,4}$ Franz X. Kärtner ${ }^{1,2,5}$ and Guoqing Chang, ${ }^{1,2^{*}}$ \\ ${ }^{1}$ Center for Free-Electron Laser Science, DESY, Notkestraße 85, 22607 Hamburg, Germany \\ ${ }^{2}$ The Hamburg Centre for Ultrafast Imaging, Luruper Chaussee 149, 22761 Hamburg, Germany \\ ${ }^{3}$ Institute of Applied Physics, Abbe center of Photonics, Friedrich-Schiller-Universität Jena, \\ Albert-Einstein-Strasse 15, 07745 Jena, Germany \\ ${ }^{4}$ Helmholtz Institut Jena, Fröbelstieg 3, 07743 Jena, Germany \\ ${ }^{5}$ Physics Department, University of Hamburg, Luruper Chaussee 149, 22761 Hamburg, Germany \\ "guoqing.chang@desy.de
}

\begin{abstract}
We demonstrate a compact Ytterbium-fiber MOPA system operating at $1018 \mathrm{~nm}$, serving as powerful and compatible seed for cryogenically-cooled Yb:YLF amplifier. The output average power reaches $87 \mathrm{~W}$ with pulse energy of $4.9 \mu \mathrm{J}$.

(일 2016 Optical Society of America

OCIS codes: (320.7080) Ultrafast Devices; (060.2320) Fiber optics amplifiers and oscillators
\end{abstract}

Yb-doped materials have attracted much attention for their high optical-to-optical efficiency enabling the generation of high energy pulses at high average power. Among them, cryogenically cooled Yb:YLF exhibiting spectral width of several-nm has shown potential achieving sub-picosecond pulse duration with several-mJ energy at $\mathrm{kHz}$ repetition rate [1]. Cryogenically cooled Yb:YLF offers a saturation fluence higher than that of cryogenically cooled $\mathrm{Yb}: Y A G$, a longer excited state lifetime, and a smaller quantum defect therefore suggesting potential for high energy extraction at high repetition rate. These attractive spectroscopic properties however require for a seed laser operating at $1018 \mathrm{~nm}$ central wavelength and with a large stretching factor to permit efficient and damage-free energy extraction in subsequent $100 \mathrm{~mJ}$-class amplifiers we are developing. In a previous implementation [1], a Ti:sapphire laser with low pulse energy and limited stretching ratio was used. A Yb-doped fiber-based, high energy seed would facilitate the implementation of a $100 \mathrm{~mJ}$ cryogenically cooled $\mathrm{Yb}: \mathrm{YLF}$ amplifier chain, mitigating ASE as well as gain-narrowing in the onwards amplifier chain and incorporating the advantage of low-cost and reliability.

Here, we demonstrate the first high power fiber-based system specifically designed to optimally seed a high energy Yb:YLF amplifier chain. The fiber-based master-oscillator-power-amplifier (MOPA) seed system operating at a repetition rate of $17.88 \mathrm{MHz}$ produces $87-\mathrm{W}, 4.9-\mu \mathrm{J}$ pulses centered at $1018 \mathrm{~nm}$ with 1-ns stretched duration and 2-nm bandwidth, ideally matching the emission spectrum of cryogenic Yb:YLF crystals.

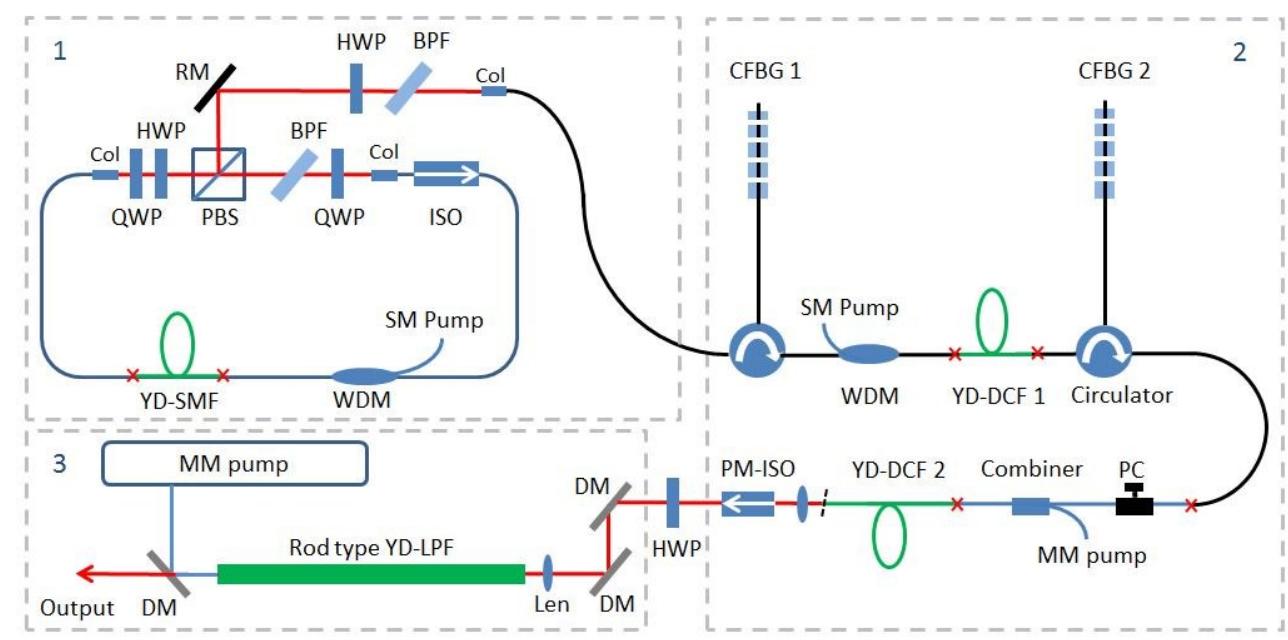

Fig. 1: Schematic of $1018 \mathrm{~nm}$ MOPA system. HWP: half-wave plate, QWP: quarter-wave plate, BPF: band-pass filter, PBS: polarization beam splitter, RM: reflecting mirror, Col: collimator, WDM: wavelength division multiplexer, SM: single mode, CFBG: chirped fiber Bragg grating, PC: polarization controller, MM: multimode, PM-ISO: polarization sensitive isolator, DM: dichroic mirror, YD: ytterbium-doped, SMF: single mode fiber, DCF: double-clad fiber, LPF: large-pitch fiber, blue line: non PM fiber, black line: PM fiber 
The experimental setup (Fig. 1) consists of three parts. Part 1 includes a 17.88-MHz oscillator mode-locked at $1020 \mathrm{~nm}$ central wavelength via an intra-cavity narrow band-pass filter (BPF) and nonlinear polarization evolution (NPE) mechanism. Another BPF - outside the cavity - selects a 2-nm wide spectral band around $1016 \mathrm{~nm}$ (Fig. 2(a) - red curve) from the broadband output of the oscillator (Fig. 2(a) - blue curve). A half-wave plate adjusts the polarization direction of the pulses coupled into the polarization maintaining (PM) fiber.

Part 2 includes two PM circulators connected to two PM chirped fiber Bragg gratings (CFBGs), which stretch the laser pulse to $\sim 1$ ns duration therefore alleviating nonlinear effects in subsequent amplifier stages. A preamplifier based on 2-m double cladding PM Yb-doped fiber is inserted between the two circulators to compensate the losses introduced by the circulators and CFBGs. Since the peak gain of this fiber amplifier is near $1030 \mathrm{~nm}$, the center wavelength of the pulses shifts from $1016 \mathrm{~nm}$ to $1018 \mathrm{~nm}$ at this stage. Spliced directly to the port 3 of circulator 2, the second amplifier consists of a $(2+1)$ beam combiner, a multi-mode pumping diode, and 1-m absorption-flattened Yb-doped double-clad fiber (FP fiber). This FP fiber provides higher gain at $1018 \mathrm{~nm}$ than 1030 $\mathrm{nm}$, and thus efficiently amplifies the signal wavelength to an average power of $4 \mathrm{~W}$. Figure 2(b) shows that the slope efficiency of FP fiber amplifier is $78 \%$. Although FP fiber is non-PM, adjusting the polarization controller at the combiner input results in $18-\mathrm{dB}$ polarization extinction ratio. The output spectrum from part 2 is shown in Fig. 2(b) (inset).

Part 3 is the power amplifier consisting on a 1.2-m Yb-doped rod-type large-pitch fiber (LPF) [2], which can produce $100 \mathrm{~W}$, high quality $\mu \mathrm{J}$ pulses configured in pre-chirp managed amplification [3]. The signal and pump are coupled into the LPF from opposite direction. Figure 2(c) shows the output spectrum versus average power of the amplified pulses. The signal spectrum remains unchanged, suggesting a linear amplification. Broadband ASE peaking at $1030 \mathrm{~nm}$ grows with the increased pump power, yet remains $26 \mathrm{~dB}$ below the signal spectral peak even at the highest average power of $87 \mathrm{~W}$. The slope efficiency of the LPF amplifier is limited to $49 \%$ (Fig. 2(d)) because the rod type fiber has its gain peaking at $1030 \mathrm{~nm}$. Inset of Fig. 2(d) shows the excellent spatial profile of the 87-W amplified pulses.
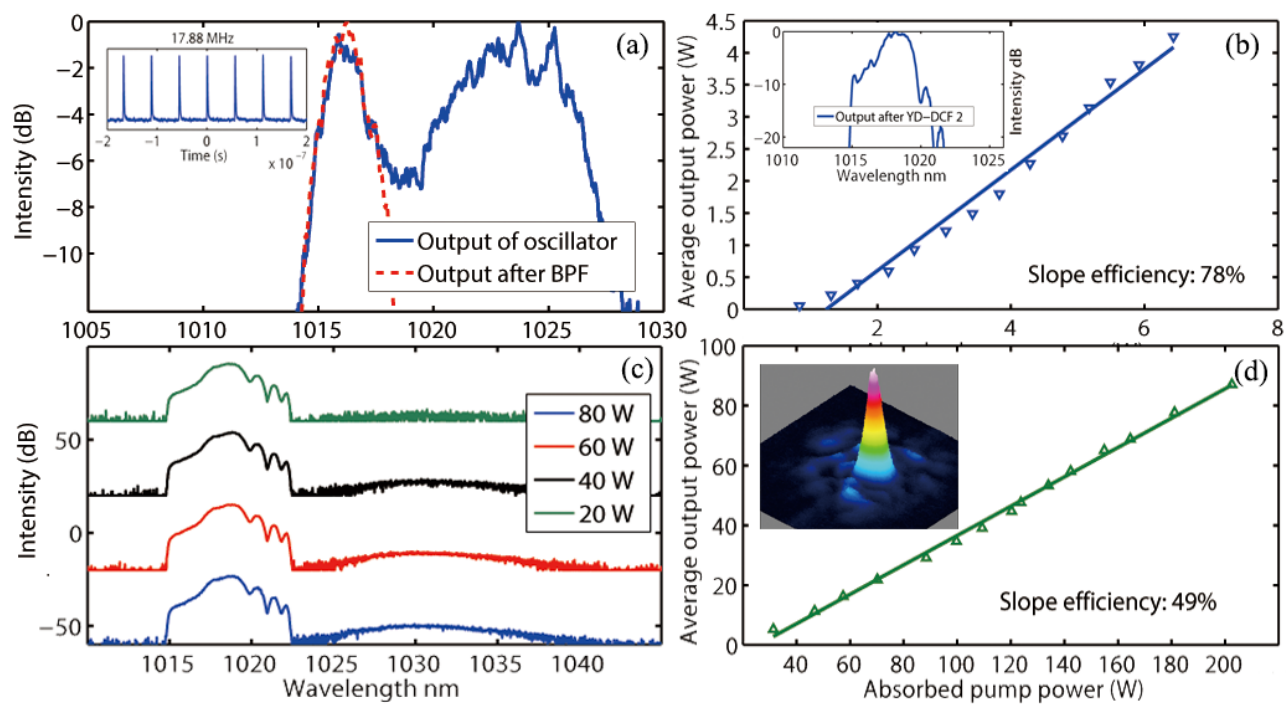

Fig. 2. (a) Measured output spectrum of oscillator (blue curve), spectrum after BPF with $2 \mathrm{~nm}$ bandwidth at $1016 \mathrm{~nm}$ (red curve) and 17.88 MHz pulse train (inset); (b) measured characteristics output power versus absorbed pump power of FP fiber amplifier and output spectrum of FP fiber at $1018 \mathrm{~nm}$ at $4 \mathrm{~W}$ power (inset); (c) measured output spectrum of rod-type LPF amplifier at $1018 \mathrm{~nm}$ for increasing pump powers; (d) measured characteristics output power versus absorbed pump power of rod-type LPF amplifier and spatial profile (inset).

In conclusion, we report a high-power Yb-fiber MOPA system operating at $1018 \mathrm{~nm}$ with $4.9 \mu \mathrm{J}$ pulse energy. With its output spectrum precisely matching the gain bandwidth of Yb:YLF crystal, this laser system serves as a desired seed source for subsequent $100 \mathrm{~mJ}$-class, sub-ps duration cryogenic Yb:YLF amplifiers.

\section{References}

[1] D. E. Miller et al., "Sub-picosecond pulses at $100 \mathrm{~W}$ average power from a Yb:YLF chirped-pulse amplification system", Opt. Lett. 37, 2700 (2012).

[2] J. Limpert et al., "Yb-doped large-pitch fibres: effective single-mode operation based on higher-order mode delocalisation," Light Sci. Appl. 1, e8 (2012).

[3] W. Liu et al., "Pre-chirp managed nonlinear amplification in fibers delivering 100 W, 60 fs pulses", Opt. Lett. 40, 151 (2015). 